\title{
Quantification of Leishmania infantum DNA in females, eggs and larvae of Rhipicephalus sanguineus
}

\author{
Filipe Dantas-Torres, Maria Stefania Latrofa and Domenico Otranto*
}

Background: Leishmania infantum is a widespread parasite that affects dogs and humans worldwide. It is transmitted primarily by phlebotomine sand flies, but recently there has been much discussion on the role of the brown dog tick, Rhipicephalus sanguineus, as a potential vector for this protozoan. Recent laboratory and field investigations have contributed to this hypothesis, but a proof of the vector capacity of $R$. sanguineus has yet to be provided. Following a recent study suggesting that $L$. infantum passes transovarially from the female tick to her progeny the current study provides new evidence of the transovarial transmission of $L$. infantum in $R$. sanguineus.

Methods: Engorged females of $R$. sanguineus were collected from the environment in a dog shelter of southern Italy, where canine leishmaniosis is endemic. In the laboratory, 97 females that successfully laid eggs, their eggs and the originated larvae were subjected to DNA extraction and then tested by a TaqMan-based real time PCR targeting a fragment of the kinetoplast DNA (kDNA) of L. infantum.

Results and conclusions: L. infantum kDNA was detected in engorged females, their eggs and originating larvae, with a parasite load ranging from $1.8 \times 10^{-4}$ to $10.0 \times 10^{0}$. Certainly, the current study provides further evidence on the passage of $L$. infantum from $R$. sanguineus females to their offspring. The observation of promastigote forms in larvae is necessary to definitively confirm this hypothesis, which would raise interesting questions about the possible role of ticks in the maintenance of $L$. infantum infection among dogs in certain areas.

\section{Background}

Leishmania parasites (Kinetoplastida: Trypanosomatidae) are digenetic protozoa responsible for a group of parasitic diseases generally referred to as the leishmaniases. These diseases, most of which are zoonoses, are responsible for a huge burden on public health, causing considerable morbidity and mortality in about 88 countries over the world $[1,2]$. Among the different clinical forms of the disease, the visceral one is of major importance for being life-threatening and for affecting mainly children and immunodepressed individuals $[1,2]$.

Leishmania infantum (synonym, Leishmania chagasi) is one of the causative agents of visceral leishmaniasis, an important zoonosis in Europe, Africa, Asia and America [1-4]. This protozoan is primarily maintained in nature by wild reservoir hosts, such as rodents,

\footnotetext{
* Correspondence: filipe.vet@globo.com; d.otranto@veterinaria.uniba.it
Dipartimento di Sanità Pubblica e Zootecnia, Università degli Studi di Bari,

* Correspondence: filipe.vet@globo.com; d.otranto@veterinaria.uniba.it
Dipartimento di Sanità Pubblica e Zootecnia, Università degli Studi di Bari, Valenzano, BA, Italy
} Valenzano, BA, ltaly

marsupials, edentates and canids [5]. In the peridomestic transmission cycle, dogs play a role as reservoir hosts for L. infantum, mainly because they are quite susceptible to the infection and present a typically heavy skin parasitism [6], which ultimately facilitates the acquisition of the parasites by phlebotomine sand fly vectors (Diptera: Psychodidae), while they are taking a bloodmeal.

Although $L$. infantum is primarily transmitted by phlebotomine sand flies [7], secondary modes of transmission (e.g., transplacental transmission and via blood transfusion) have been claimed to exist [8-10]. Recently, it has been demonstrated that day-feeding midges (Diptera: Ceratopogonidae) of the genus Forcipomyia can support the development of an undescribed species of Leishmania that was originally detected in red kangaroos (Macropus rufus) in Australia around eight years ago [11]. Moreover, there has long been speculation about the role of fleas and ticks as vectors of L. infantum [12] and recent studies have reinforced this 
hypothesis $[13,14]$. Nonetheless, a definitive proof that fleas or ticks can efficiently transmit $L$. infantum from dog to dog under natural conditions has yet to be provided [15].

In a recent study, L. infantum kinetoplast DNA (kDNA) was detected in eggs and larvae from infected females, even four months post-inoculation, suggesting the possibility of transovarial passage of the protozoa in $R$. sanguineus [16]. However, the aforementioned study was performed using experimentally infected females, which were artificially inoculated with stationary-phase promastigotes [16]. Undoubtedly, it would be valuable to reassess this hypothesis using naturally infected females. In this perspective, the present investigation was carried out in order to demonstrate the occurrence of transovarial passage of L. infantum kDNA in naturally infected $R$. sanguineus ticks. In particular, the research's specific objectives were to detect and quantify the amount of $L$. infantum kDNA present in engorged, wild-collected females, their laid eggs and the originating larvae, using a highly sensitive real time polymerase chain reaction (PCR) protocol.

\section{Methods}

\section{Collection, identification and rearing of ticks}

On 30 April 2009, engorged female ticks $(n=100)$ were collected directly from the environment in a dog shelter located in southern Italy, where $R$. sanguineus is the only tick species present [17]. In the laboratory, the females were rinsed in distilled water and dried with a clean filter paper. The identity of the ticks was immediately determined based on morphology [18].

The engorged females were placed in individual plastic collectors with some holes on the top for allow the air to enter. Then, the vials were placed in an incubator under controlled conditions $\left(26 \pm 1^{\circ} \mathrm{C}, 70 \pm 10 \%\right.$ relative humidity, and scotophase) for the females to lay their eggs. Each female was monitored every day and, after the end of the oviposition period, they and their egg batches $(\sim 10 \mathrm{mg})$ were separated for subsequent DNA extraction, being the remaining eggs left in the incubator. After the eggs were hatched, larvae from 16 females were separated in 10 pools of 10 larvae each. As a rule, females, eggs and larvae were frozen at $-20^{\circ} \mathrm{C}$ until DNA extraction.

\section{DNA extraction and real time PCR protocol}

Genomic DNA was extracted from 97 females (3 females did not lay eggs), eggs and larvae using DNeasy Blood \& Tissue Kit (Qiagen, GmbH, Hilden, Germany), in accordance with the manufacturer's instructions. Additionally, and differently from the extraction protocol, all tick specimens were pre-treated by three cycles of freezing $\left(-80^{\circ} \mathrm{C}\right)$ and boiling for $10 \mathrm{~min}$, and the extracted DNA was eluted in $50 \mu \mathrm{l}$ of elution buffer $\mathrm{AE}$ (Qiagen).

Real-time PCR for simultaneous detection and quantification of $L$. infantum kinetoplast minicircle DNA was performed using primers LEISH-1 (5'-AACTTTTCTGG TCCTCCGGGTAG-3') and LEISH-2 (5'-ACCCCCAG TTTCCCGCC-3') and TaqMan-MGB probe (FAM5'-AAAAATGGGTGCAGAAAT-3'-non-fluorescent quencher-MGB) designed by Francino and collaborators [19]. The reaction mixture $(20 \mu \mathrm{l})$ contained $10 \mu \mathrm{l}$ of $\mathrm{iQ}^{\mathrm{TM}}$ Supermix (Bio-Rad Laboratories, Hercules CA, USA), each primer at a concentration of $900 \mathrm{nM}$, the probe at a concentration of $200 \mathrm{nM}$, and $2 \mu \mathrm{l}$ of template DNA. The run consisted of a hot start at $95^{\circ} \mathrm{C}$ for $3 \mathrm{~min}$ and 42 cycles of denaturation $\left(95^{\circ} \mathrm{C}\right.$ for $\left.10 \mathrm{sec}\right)$ and annealing-extension $\left(60^{\circ} \mathrm{C}\right.$ for $\left.30 \mathrm{sec}\right)$. All assays were carried out in duplicate, with negative $(R$. sanguineus from a laboratory colony) and positive (lymph node tissue from a $L$. infantum-infected dog) controls included in each run. The GPCR was performed in a CFX96 ${ }^{\mathrm{TM}}$ Real-Time System (Bio-Rad Laboratories, Inc., Hercules CA, USA). The increase in the fluorescent signal was registered during the extension step of the reaction and the data analysed by CFX Manager ${ }^{\mathrm{TM}}$ Software Version 1.6 (Bio-Rad).

Parasites were quantified by the absolute quantification method. A 10-fold dilution series of standard DNA from promastigotes (log phase concentration, $1.7 \times 10^{6}$ parasites $/ \mathrm{ml}$ ) of L. infantum (zymodeme MON-1) was used as calibrators, allowing plotting a standard curve, each dilution being tested in triplicate. The limit of detection of the real time PCR was assessed using a serial dilution from $1.7 \times 10^{-1}$ to $1.7 \times 10^{-8}$ parasites per reaction. The inter-assay reproducibility was estimated by testing 10 -fold serial dilutions, being the experiment repeated 10 times. Results were expressed as the number of parasites per reaction mixture (i.e., $2 \mu \mathrm{l}$ of template DNA), taking into account the initial concentration and subsequent dilutions used to plot the standard curve.

\section{Statistical analysis}

Positivity rates were calculated as the number of positive samples divided by the number of tested samples multiplied by 100, and expressed as percentages. Additionally, the minimum (number of positive pools/total number of larvae tested) and maximum (number of positive pools/ number of larvae in positive pools) positivity rates were calculated for larvae, considering that at least one larva in each positive pool must be positive. Ninety five percent confidence intervals ( $95 \% \mathrm{CI}$ ) were calculated for each positivity rate. Again, the parasitic load (number of parasites per reaction mixture) in females and eggs were compared using Mann-Whitney U test, differences being 
considered statistically significant when $P$ was 0.05 or less $(P \leq 0.05)$. Finally, the parasite loads detected in females and eggs were statistically compared using the Spearman's rank correlation coefficient (rs). Statistical analyses were carried out using BioEstat (version 5.0; Mamirauá/CNPq, Belém, PA, Brazil).

\section{Results}

Out of 97 field-collected engorged females of R. sanguineus tested by real time PCR for the detection of L. infantum kDNA, 25 (25.8\%, 95\% CI: 17.1\%-34.5\%) were positive, with a parasite load (mean \pm standard deviation: $3.4 \times 10^{-1} \pm 7.7 \times 10^{-1}$ ) ranging from $2.9 \times$ $10^{-3}$ to $2.8 \times 10^{0}$ parasites per PCR reaction. In the same way, out of 97 pools of eggs laid by the aforementioned females, 39 (40.2\%, 95\% CI: 30.5\%-50.0\%) were positive by real time PCR, with a parasite load $\left(2.8 \times 10^{-}\right.$ ${ }^{1} \pm 1.6 \times 10^{0}$ ) ranging from $1.3 \times 10^{-4}$ to $10.0 \times 10^{0}$ parasites per PCR reaction.

Out of 160 pools of larvae tested, 93 (58.1\%, 95\% CI: $50.5 \%-65.8 \%)$ were positive, with a parasite load $(2.9 \times$ $10^{-3} \pm 1.0 \times 10^{-2}$ ) ranging from $1.8 \times 10^{-4}$ to $9.8 \times 10^{-2}$ parasites per PCR reaction. The overall minimum and maximum positivity rates were $5.9 \%$ (95\% CI: $4.7 \%-7 \%$ ) and $10 \%$ (95\% CI: 8.1\%-11.9\%). In particular, among the 93 positive pools of larvae, 56 (60.2\%, 95\% CI: $50.3 \%$ $70.2 \%)$ were from eight positive females and 37 (39.8\%, 95\% CI: 29.8\%-49.7\%) from eight negative females. Furthermore, 58 positive pools of larvae came from 10 positive pools of eggs whereas 35 positive pools of larvae came from six negative pools of eggs.

No statistically significant difference was found when the parasite loads detected in females and in pools of eggs were compared (Mann-Whitney $\mathrm{U}$ test, $P=0.706$ ). In the same way, no significant correlation was found between the parasite load detected in females and pools of their eggs $(r s=-0.03, P=0.718)$.

The real time-PCR protocol used in this study was able to detect very low amounts of L. infantum kDNA, with a limit of detection of $1.7 \times 10^{-6}$ parasites per PCR reaction (data not shown). Figure 1 presents the standard curve, slope and efficacy of a typical experiment. The mean Ct (cycle threshold) values for positive females, eggs and larvae were 35, 36 and 36, respectively.

\section{Discussion}

The present study demonstrates that $L$. infantum kDNA is passed from the engorged female tick to her originating larvae. Because no statistically significant differences were found in relation to the estimated numbers of parasites detected by real time PCR in females and their eggs, it appears that the amount of L. infantum kDNA and, most likely, the number of parasites present in

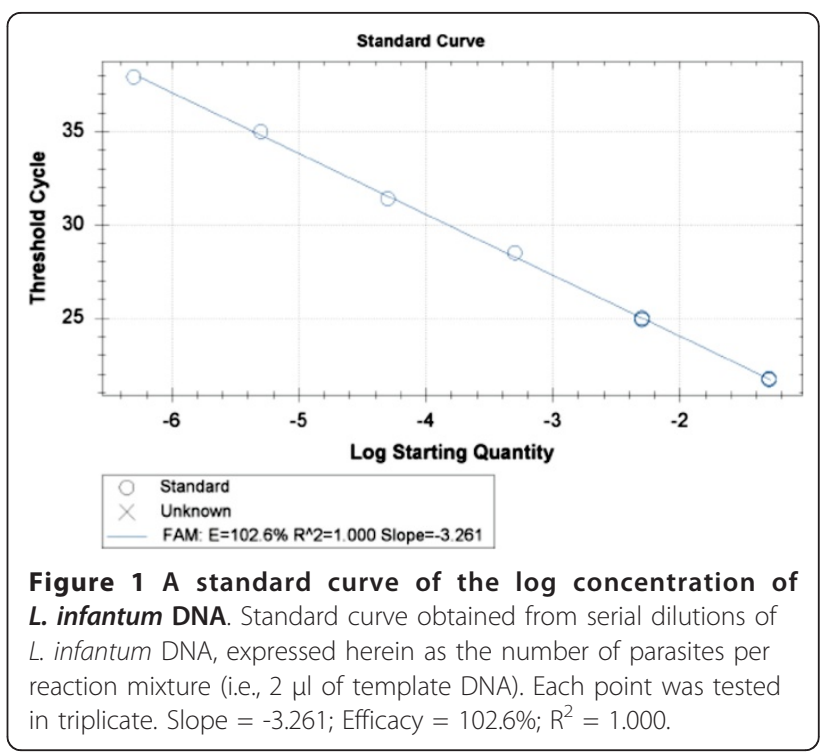

females, remained relatively stable in her progeny. This finding was remarkable, especially if one considers that only a small amount of the eggs from each female was tested by real time PCR. This suggests that the amount of $L$. infantum kDNA would probably be greater if more eggs had been tested.

Concerning the correlation between the positivity of engorged females, eggs and larvae, different situations were documented: (I) female positive $\rightarrow$ eggs positive $\rightarrow$ larvae positive; (II) female positive $\rightarrow$ eggs negative $\rightarrow$ larvae positive; (III) female negative $\rightarrow$ eggs positive $\rightarrow$ larvae positive; (IV) female negative $\rightarrow$ eggs negative $\rightarrow$ larvae positive; and (V) female negative $\rightarrow$ eggs negative $\rightarrow$ larvae negative (data not shown). The absence of correlation between the positivity in engorged females, eggs and larvae might be explained by the presence of PCR inhibitors in engorged females (e.g., situations III and IV) and by the fact that not all eggs or larvae were tested by real time PCR. Thus, in the cases II and IV, it is probable that eggs and/or larvae had been positive if additional samples of eggs and larvae had been tested. Although minimal, the possibility of contamination in one of these egg and larval pools cannot be completely ruled out.

An early study conducted in France failed to demonstrate the occurrence of transovarial passage of $L$. infantum in $R$. sanguineus [20]. More recently, an experimental study using a high sensitive real time-PCR protocol reported the detection of kDNA in artificially infected engorged females, their eggs and the originating larvae [16]. These findings are corroborated by the present study, which included field-collected females that had fed on dogs naturally infected by L. infantum instead of artificially infected engorged females used in the previous study [16]. As such, these results reinforce 
the hypothesis of the occurrence of transovarial passage of L. infantum in $R$. sanguineus. The confirmation of this theory would provide a strong supporting evidence for the participation of $R$. sanguineus in the maintenance of $L$. infantum in nature.

Further research to assess the presence and viability of L. infantum in unfed larvae is needed in order to fully assess the occurrence of transovarial transmission of $L$. infantum in $R$. sanguineus. Indeed, this hypothesis needs to be confirmed by the visualization of promastigotes in unfed larvae or by the detection of L. infantum RNA expression, in order to substantiate the present of live parasites in ticks. Accordingly, L. infantum RNA has recently been detected in ticks collected from naturally infected dogs [21], confirming definitively the viability of the parasites in the ticks and supporting the participation of $R$. sanguineus as an invertebrate host of $L$. infantum. In fact, $R$. sanguineus is a competent definitive host for many pathogens, including the protozoa Babesia vogeli and Hepatozoon canis [22].

In conclusion, the present investigation reports the detection and quantification of L. infantum DNA in field-collected engorged females, eggs and larvae, providing further evidence on the occurrence of transovarial passage of L. infantum in $R$. sanguineus, although this hypothesis has yet to be proven. This finding suggests that the hypothesis of ticks as vectors of this protozoan between dogs is reasonable and needs to be finally confirmed by finding of promastigote forms in these arthropods.

\footnotetext{
Acknowledgements

Thanks to Dr. Stefania Weigl for her help with some laboratory activities. This research and the publication of the thematic series have been sponsored by Bayer Animal Health $\mathrm{GmbH}$.
}

\section{Authors' contributions}

FDT collected, identified and reared the ticks in the laboratory, contributed with data analysis and interpretation and wrote the first draft of the manuscript. MSL run the real time PCRs and contributed with data analysis and interpretation. DO contributed with data analysis and interpretation and revision of the manuscript. All authors read and approved the final version of the manuscript.

\section{Competing interests}

The authors declare that they have no competing interests.

Received: 16 January 2011 Accepted: 13 April 2011

Published: 13 April 2011

\section{References}

1. Desjeux P: Leishmaniasis: current situation and new perspectives. Comp Immunol Microbiol Infect Dis 2004, 27:305-318.

2. Bern C, Maguire JH, Alvar J: Complexities of assessing the disease burden attributable to leishmaniasis. PLoS Negl Trop Dis 2008, 2:e313.

3. Baneth G, Koutinas AF, Solano-Gallego L, Bourdeau P, Ferrer L: Canine leishmaniosis - new concepts and insights on an expanding zoonosis: part one. Trends Parasitol 2008, 24:324-330.

4. Dantas-Torres F: Canine leishmaniosis in South America. Parasit Vectors 2009, 2(Suppl 1):S1.
5. Ashford RW: Leishmaniasis reservoirs and their significance in control. Clin Dermatol 1996, 14:523-532.

6. Dantas-Torres F: The role of dogs as reservoirs of Leishmania parasites, with emphasis on Leishmania (Leishmania) infantum and Leishmania (Viannia) braziliensis. Vet Parasitol 2007, 149:139-146.

7. Killick-Kendrick R: Phlebotomine vectors of the leishmaniases: a review. Med Vet Entomol 1990, 4:1-24.

8. Rosypal AC, Troy GC, Zajac AM, Frank G, Lindsay DS: Transplacental transmission of a North American isolate of Leishmania infantum in an experimentally infected beagle. J Parasitol 2005, 91:970-972.

9. Freitas E, Melo MN, Costa-Val AP, Michalick MS: Transmission of Leishmania infantum via blood transfusion in dogs: potential for infection and importance of clinical factors. Vet Parasitol 2006, 137:159-167.

10. Silva FL, Oliveira RG, Silva TM, Xavier MN, Nascimento EF, Santos RL: Venereal transmission of canine visceral leishmaniasis. Vet Parasitol 2009, 160:55-59.

11. Dougall AM, Alexander B, Holt DC, Harris T, Sultan AH, Bates PA, Rose K, Walton SF: Evidence incriminating midges (Diptera: Ceratopogonidae) as potential vectors of Leishmania in Australia. Int J Parasitol 2011, 41:571-579.

12. Wenyon CM: The transmission of leishmania infections: A review. Trans Roy Soc Trop Med Hyg 1932, 25:319-351.

13. Coutinho MT, Bueno LL, Sterzik A, Fujiwara RT, Botelho JR, Maria M, Genaro O, Linardi PM: Participation of Rhipicephalus sanguineus (Acari: Ixodidae) in the epidemiology of canine visceral leishmaniasis. Vet Parasitol 2005, 128:149-155.

14. Coutinho MT, Linardi PM: Can fleas from dogs infected with canine visceral leishmaniasis transfer the infection to other mammals? Vet Parasitol 2007, 147:320-325

15. Dantas-Torres F: Ticks as vectors of Leishmania parasites. Trends Parasitol 2011, 27:155-159.

16. Dantas-Torres F, Martins TF, de Paiva-Cavalcanti M, Figueredo LA, Lima BS, Brandão-Filho SP: Transovarial passage of Leishmania infantum kDNA in artificially infected Rhipicephalus sanguineus. Exp Parasitol 2010, 125:184-185

17. Lorusso V, Dantas-Torres F, Lia RP, Tarallo VD, Mencke N, Capelli G, Otranto D: Seasonal dynamics of the brown dog tick (Rhipicephalus sanguineus) on a confined dog population in Italy. Med Vet Entomol 2010, 24:309-315.

18. Walker JB, Keirans JE, Horak IG: Genus Rhipicephalus (Acari, Ixodidae). A guide to the brown ticks of the world. Cambridge: Cambridge University Press; 2000.

19. Francino O, Altet L, Sánchez-Robert E, Rodriguez A, Solano-Gallego L, Alberola J, Ferrer L, Sánchez A, Roura X: Advantages of real-time PCR assay for diagnosis and monitoring of canine leishmaniosis. Vet Parasitol 2006, 137:214-221

20. Blanc $G$, Caminopetros J: La transmission du Kala-Azar méditerranéen par une tique: Rhipicephalus sanguineus. C R Acad Sci 1930, 191:1162-4.

21. Colombo FA, Odorizzi RMFN, Laurenti MD, Galati EAB, Canavez F, PereiraChioccola VL: Detection of Leishmania (Leishmania) infantum RNA in fleas and ticks collected from naturally infected dogs. Parasitol Res

22. Dantas-Torres F: The brown dog tick, Rhipicephalus sanguineus (Latreille, 1806) (Acari: Ixodidae): from taxonomy to control. Vet Parasitol 2008, 152:173-185.

doi:10.1186/1756-3305-4-56

Cite this article as: Dantas-Torres et al:: Quantification of Leishmania infantum DNA in females, eggs and larvae of Rhipicephalus sanguineus. Parasites \& Vectors 2011 4:56. 